Binghamton University

The Open Repository @ Binghamton (The ORB)

\title{
Lateral pull-in instability of electrostatic MEMS transducers employing repulsive force
}

Meysam Daeichin

Binghamton University--SUNY, mdaeich1@binghamton.edu

Ronald Miles

Binghamton University--SUNY, rmiles@binghamton.edu

Shahrzad Towfighian

Binghamton University--SUNY, stowfigh@binghamton.edu

Follow this and additional works at: https://orb.binghamton.edu/mechanical_fac

Part of the Mechanical Engineering Commons

\section{Recommended Citation}

Daeichin, Meysam; Miles, Ronald; and Towfighian, Shahrzad, "Lateral pull-in instability of electrostatic MEMS transducers employing repulsive force" (2020). Mechanical Engineering Faculty Scholarship. 30. https://orb.binghamton.edu/mechanical_fac/30

This Article is brought to you for free and open access by the Mechanical Engineering at The Open Repository @ Binghamton (The ORB). It has been accepted for inclusion in Mechanical Engineering Faculty Scholarship by an authorized administrator of The Open Repository @ Binghamton (The ORB). For more information, please contact ORB@binghamton.edu. 


\title{
Lateral pull-in instability of electrostatic MEMS transducers employing repulsive force
}

\author{
Meysam Daeichin - Ronald Miles - Shahrzad Towfighian
}

Received: date / Accepted: date

\begin{abstract}
We report on the lateral pull-in in capacitive MEMS transducers that employ a repulsive electrostatic force. The moving element in this system undergoes motion in two dimensions. A two degree-offreedom mathematical model is developed to investigate the pull-in quantitatively. The nonlinear electrostatic force, which is a vector function of two spatial coordinates, is determined by calculating the potential energy of the system using a boundary element approach. The equilibrium points are found by numerically solving the nonlinear coupled static equations. A stability analysis reveals that depending on the values of the lateral and transverse stiffness, the system undergoes different bifurcations when the voltage on the side electrodes is considered as the control parameter. Three-dimensional bifurcation diagrams are presented and discussed to elucidate the nonlinear nature of the system. The results establish important criteria for designing MEMS transducers with reliable and robust performance.
\end{abstract}

Keywords Electrostatic - Pull-in - Bifurcation . Stability Analysis - Repulsive Force - MEMS . Instability

M. Daeichin · Ronald Miles · Shahrzad Towfighian State University of New York at Binghamton, 4400 Vestal Parkway East, Binghamton, NY 13902

M. Daeichin

mdaeich1@binghamton.edu

S. Towfighian (Corresponding Author) stowfigh@binghamton.edu

R. Miles

miles@binghamton.edu

\section{Introduction}

The robust performance of capacitive MEMS (MicroElectro-Mechanical-Systems) transducers such as accelerometers $[1,2]$, gyroscopes $[3,4]$, pressure sensors $[5$, $6]$, microphones $[7,8]$, and switches [9] is crucial to the reliable operation of many devices we use on a daily basis such as our cars and smart-phones. Therefore, identifying and investigating the failure cause(s) of MEMS transducers is an important step toward creating functional devices.

There are different modes of mechanical failures for capacitive MEMS devices [10]. For example, buckling [11], snap-through [12] and pull-in instability [13,14], jeopardize the reliable performance of these devices. In general, the cause of failure for a MEMS device should be eliminated in the design process when possible [15], or be controlled and mitigated $[16,17]$.

In this study, we report on the lateral instability of electrostatic MEMS transducers that are based on the repulsive force. Repulsive force-based transducers were introduced to address the pull-in problem inherent in conventional capacitive transducers that utilize two conductors $[18,19]$. These two-conductor transducers come in different design flavors, but they mainly fall into two categories, parallel plates [20] and comb drive [4] configurations. The moving structure in the twoconductor design can be flexible, such as a flexible microbeam [21-23] above an underlying rigid electrode, or the moving element can be rigid, suspended with compliant elements above a fixed electrode or substrate [24].

In two-conductor transducers, pull-in happens when the deflection of the moving electrode exceeds a certain 


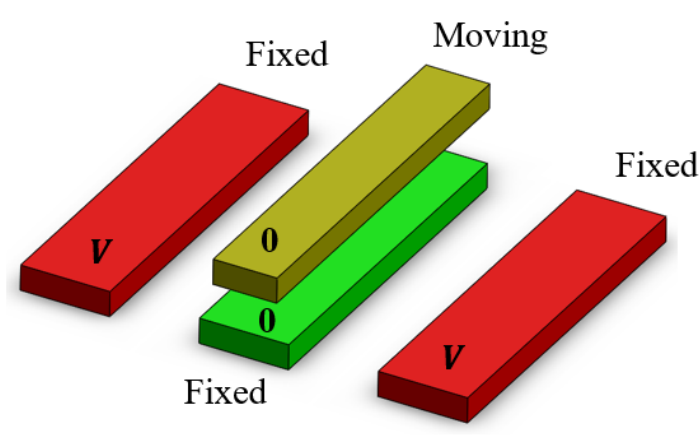

(a)

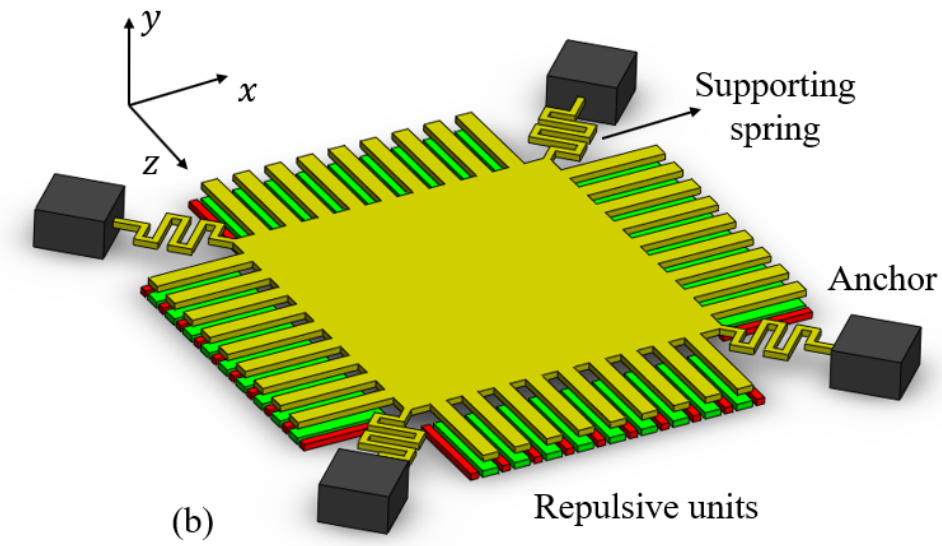

(b)

Fig. 1: (a) A repulsive electrode set (b) The schematic of the microstructure with repulsive electrode sets on its four sides. The number of the electrode sets on each side is not equal to the number of the electrode sets in the real microstructure and this is for presentation only.

threshold and goes close to the other electrode (often one-third of the initial gap between them) [25]. In this situation, because the two electrodes have different voltages, the attractive electrostatic force increases dramatically, and the restoring force of the supporting elements cannot keep the microstructure stable. As a result, the moving electrode collapses to the fixed electrode and gets stuck to it. This often leads to permanent failure of the microstructure. A comprehensive review of the failure of MEMS devices because of pull-in instability is given in [26].

Pull-in occurs in static and dynamic modes. Static pull-in could be triggered by increasing the DC voltage between the two conductors. This phenomenon could be beneficial in applications such as triggering a switch [27] in an electrical circuit or be catastrophic in actuators such as micro-mirrors [28]. There are numerous studies in the literature investigating the static pull-in in MEMS transducers [13,24,26,29]. Dynamic pull-in usually happens before the threshold for the static pullin is passed [30]. The dynamic pull-in could be triggered by different factors such as damping conditions [31] and time-varying electrostatic or mechanical loads [30,32]. The dynamic pull-in could also be advantageous [32] or catastrophic [26]. There are numerous studies in the literature, analyzing dynamic pull-in instability [26,30$32]$.

On the other hand, in the Repulsive-based approach, there are three types of electrodes (Fig. 1 (a)). One is the moving grounded electrode, one is the fixed grounded electrode, and the last type is two fixed electrodes that carry voltage and are placed to the side of the grounded fixed electrode [33]. We call these three types of elec- trodes moving electrode, bottom electrode, and side electrodes, respectively. With this configuration and up to a certain gap between the moving and bottom electrodes, the electrostatic force pushes the moving electrode away from the bottom electrode [34]. After the certain gap is passed, the electrostatic force changes its sign and attracts the moving electrode to the bottom electrode. This nature of the electrostatic force eliminates the possibility of the moving electrode being pulled into the bottom electrode. In fact, even if the two electrodes come into contact, they would not get stuck to each other because they both carry the same voltage $[35,36]$. For a comprehensive explanation of this mechanism, see $[33,35]$.

Although this design approach successfully eliminates the pull-in possibility between the moving and bottom electrodes [15], we have observed experimentally that pull-in could happen between the moving and side electrodes. There are numerous studies in the literature where the Repulsive-force paradigm is used in which the voltage on the side electrodes is increased to voltage levels up to two orders of magnitudes higher than voltage levels in conventional two-conductor capacitors [33,35-37]. This ability to increase the voltage without jeopardizing the device stability is extremely useful when a MEMS actuator such as a micromirror is needed with a larger range of displacement or when higher sensitivity is desired for a MEMS sensor such as microphones or accelerometers. In the Repulsive-based transducers, however, we have noticed that by increasing the voltage on the side electrodes, the microstructure will eventually pull-in to the side electrodes. 
In this study, we have constructed a two degreesof-freedom (DOF) mathematical model to investigate the static pull-in of these repulsive-based transducers. The role of the ratio between the lateral to transverse stiffness in pull-in is analyzed.
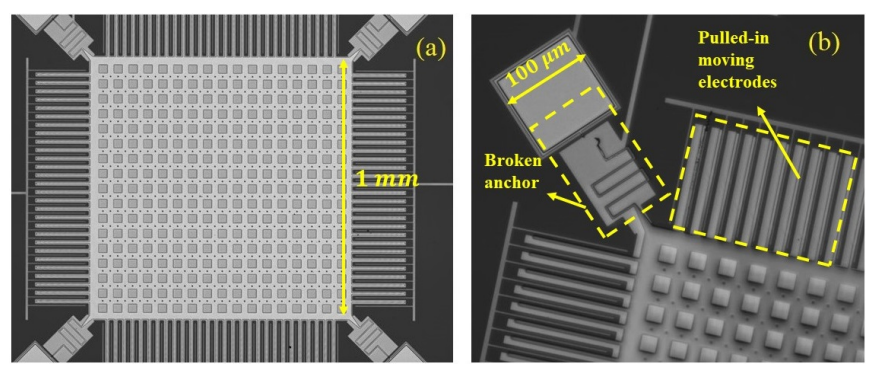

Fig. 2: (a) Fabricated microstructure (b) broken microstructure because of pull-in

The rest of this paper is organized as follows. In Section 2, the pull-in mechanism in a repulsive electrode set is examined qualitatively. In Section 3, a mathematical model with two degrees of freedom is developed to analyze the stability of the microstructure and pull-in threshold quantitatively. In Section 4, the components of the electrostatic force vector on the moving electrode are obtained by calculating the electrical potential energy of the system. Section 5 presents the stability analysis where the Jacobian matrix is constructed and its eigenvalues are extracted. The pull-in voltage for different lateral and transverse stiffness scenarios is obtained and discussed by analyzing these eigenvalues. In section 6 we have investigated the effect of fabrication imperfection on the pull-in threshold. Specifically, we have performed the stability analysis for the case where the moving electrode is not exactly at the center location above the bottom electrode. Finally, the conclusions are given in Section 7.

\section{Description of Side Instability in Repulsive-force Electrostatic Devices}

In this section, the mechanism for the pull-in instability in repulsive force-based transducers is explained qualitatively. This qualitative description helps develop intuition to understand the inherent side instability in this transduction paradigm, which becomes useful in the next section where we investigate it quantitatively.

Figure 1 shows the schematic of the microstructure we have used as an example in our study. This microstructure consists of a large plate that employs repulsive electrode sets on its four sides. The plate is suspended with four serpentine springs. The actual microstructure is shown in Fig. 2 (a). For this microstructure, we increased the voltage slowly on the side electrodes and saw that by passing $120(\mathrm{~V})$ the microstructure collapsed to the side electrodes, which led to breaking one of its supporting springs (Fig. 2 (b)). We have observed this breaking of supporting springs and collapsing of the microstructure to the side in several other incidents.

The microstructure shown in Fig. 2 (a) has 28 units of repulsive electrode sets on each side. To understand the pull-in mechanism, we examine one of these sets, which is shown in Fig. 1 (a). In each electrode set, the side electrodes carry voltage $V$ and the moving and bottom electrodes are grounded. Figure 3 shows the moving electrode in different positions relative to the other three fixed electrodes. When the moving electrode is centered above the bottom electrode (a), the vertical component of the electrostatic force at the upper surface is larger than the same component on the lower surface. This is because the bottom electrode shields the lower surface of the moving electrode. Therefore, there are fewer electric field lines that go from the side electrodes to the lower surface of the moving electrode compared to the number of lines that go to the top surface ([33]). As a result, the upward force $\left(F_{u}\right)$ is larger than the downward force $\left(F_{d}\right)$. The moving electrode is then pushed away from the bottom electrode.

Because of the upward electrostatic force, the moving electrode tends to move upward to the point where the shielding effect of the bottom electrodes becomes negligible and therefore the upward and downward electrostatic force have the same strength (b). At this point, if the moving electrode goes upward, the vertical component of the net electrostatic force becomes downward (c), trying to bring the moving electrode down to where the force is zero. And if the electrode goes downward, the electrostatic force pushes it in the upward direction again to the point where the electrostatic force is zero. This is a recipe for a stable equilibrium point.

At the same initial position for the moving electrode, the horizontal component of the electrostatic force is zero. This is because the moving electrode is at an equal distance from the two side electrodes. In other words, the horizontal electrostatic force from the rightside electrode $\left(F_{r}\right)$ is equal to the horizontal force from the left-side electrode $\left(F_{l}\right)$.

If the moving electrode is slightly perturbed to the right (e), or if it is initially displaced from its center 


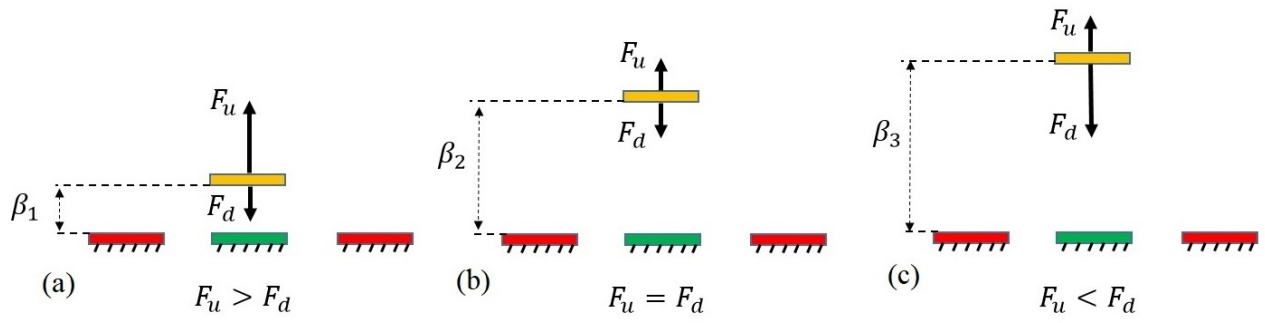

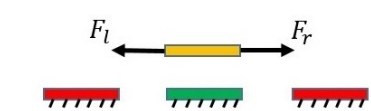

(d)

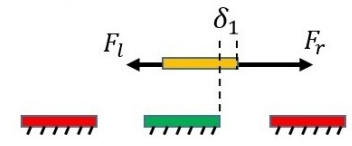

(e)

$F_{r}>F_{l}$

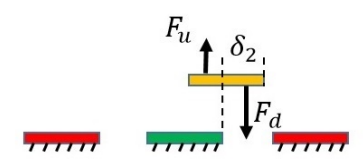

(f)

$F_{u}<F_{d}$

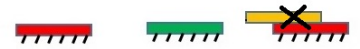

(g)

Fig. 3: Qualitative description of the pull-in in a repulsive-based electrode set. (a),(b), and (c): The change in the vertical components of the electrostatic force on the top and bottom surface of the moving electrode at different vertical gaps above the bottom electrode. (d), (e), (f), and (g): The change in the horizontal components of the electrostatic force on the moving electrode when the moving electrode moves in the lateral direction.

position, then there is an imbalance between the right and left electrostatic force $\left(F_{r}>F_{l}\right)$ and therefore, the moving electrode moves to the right. This movement to the right causes more imbalance in the rightward and leftward forces, leading to more movement to the right. This is a situation that makes the equilibrium position at center to be an unstable equilibrium point. As the moving electrode moves to the right, the force in the vertical direction changes as well. At some point, the downward force becomes larger than the upward force (f) because the shielding of the bottom electrode is no longer in effect. At that point, the moving electrode starts to move downward while it is going to the right. This means the moving electrode is headed to collapse with the side electrode. In other words, the moving electrode is pulled into the side electrode (g). This pull-in causes the failure of the microstructure.

In a real microstructure with linear restoring force in both vertical and horizontal directions, the vertical equilibrium point moves downward. This is because the restoring force can compensate for an imbalance between the upward and downward forces. So, the moving electrode does not need to go to the point where the electrostatic forces are equal. In the horizontal direction, the restoring force of the microstructure can resist the imbalance between the horizontal forces up to a certain threshold point. After this threshold point, the linear restoring force cannot keep increasing with the same rate as the electrostatic force and therefore, the pull-in will happen. As the voltage on the side electrode increases, this threshold goes closer to the center position. At a certain voltage this unstable point reaches to the center and turn the center position to an unstable equilibrium point. This voltage is the static pull-in voltage for the microstructure that establishes a maximum theoretical limit for static operation of repulsive-force devices. In the next section, we develop a mathematical model to explore how the increase in the voltage on the side electrodes leads to the pull-in of the microstructure. We construct a two DOFs mathematical model that simulates the motion of the microstructure in vertical $(y)$ and lateral directions $(x)$.

\section{Mathematical Modeling}

To model the microstructure's qualitative behavior that was explained in the previous section, a two DOF mathematical model is developed. Figure 4 illustrates this model. This figure depicts the electrode set of the microstructure shown in Fig. 1. The moving electrode is at an initial distance above the bottom electrode at the center horizontal position. The total stiffness of the serpentine springs in the lateral and vertical directions are denoted by $K_{x}$ and $K_{y}$ respectively. A lumped parameter model with two degrees of freedom is considered in Eq. (15) to simulate the motion of the microstructure in the horizontal $x$ and vertical directions $y$. In this equation, the electrostatic force components are $F_{x}(x, y)$ in the $x$ direction and $F_{y}(x, y)$ in the $y$ direction. The two functions describe the forces being exerted upon each moving electrode at point $(x, y)$ when the voltage on the side electrodes is $1(V)$. Because there are several electrode sets on the sides of the microstructure as shown in Fig. 1, the electrostatic force in the $y$ direction 


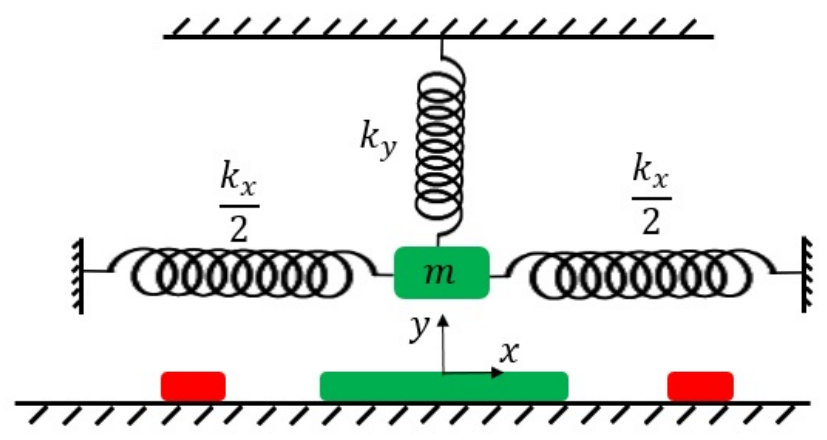

Fig. 4: Two-DOF mathematical model of the microstructure.

is multiplied by the total number of sets $(N)$. On the other hand, when the microstructure is displaced in the $x$ direction, the electrode sets on only two sides of the microstructure experience an imbalance in the electrostatic force in the lateral direction. This is why the electrostatic force in the $x$ direction is multiplied by $\frac{N}{2}$. In Eq. (15) $d$ represents the initial fabrication gap between moving and bottom electrodes. The stiffness of the microstructure in the vertical direction is calculated by measuring its natural frequency experimentally while there is no voltage on the side electrodes. This natural frequency can be used along with the mass of the microstructure to calculate the stiffness in the vertical direction. For the lateral direction, we will perform a parameter sweep on $k_{x}$ to solve the equations for different cases. We will also solve the Eq. (15) for different $k_{y}$ values to shed light on the dynamics of the system. All the other parameters in Eq. (15) are given in Table 1. It is worth mentioning that because of the symmetry in the $x$ and $z$ directions, the analysis for the $x$ direction will hold true for the $z$ direction as well. This means that as we extract the pull-in conditions from this model, the pull-in might happen in either the $x$ or $z$ direction.

$$
\left\{\begin{array}{l}
m \ddot{x}+k_{x} x=\left(\frac{N}{2}\right) V^{2} F_{x}(x, y) \\
m \ddot{y}+k_{y}(y-d)=(N) V^{2} F_{y}(x, y)
\end{array}\right.
$$

\section{Electrostatic Analysis}

In this section, the electrostatic force on the moving electrode is obtained. This electrostatic force will then be used in Eq. (15) to find the equilibrium points of the system. To find the electrostatic force, first we calculate the electrical potential energy that is stored in each electrode set when the moving electrode is at $(x, y)$. We compute the potential energy based on the boundary element approach that is explained in [38]. In this approach, each conductor's surface is divided into elements with unknown charge distribution density. Because the electrodes are maintained at constant voltages, the voltage on each element is known. Figure 5 shows this discretization along all four sides of each electrode schematically. For calculating the potential energy, we have used 200 elements on each horizontal side of the electrodes and 40 elements on each vertical side of the electrodes. The length of each element is denoted by $L_{i}$. Therefore, the amount of charge on each element can be obtained by Eq. (2).

$q_{i}=C_{i j} v_{j}$

Where $C_{i j}$ is the capacitance matrix representing the capacitance between each pair of elements. If we denote the inverse of the capacitance matrix by $G_{i j}$, then the components of this matrix can be calculated based on the geometry of the elements and their relative position as the following,

$G_{i j}=C_{i j}^{-1}\left\{\begin{array}{l}\approx-\frac{\log \left(R_{i j}\right)}{2 \pi \epsilon}(i \neq j) \\ =-\frac{1}{2 \pi \epsilon} \log \left(L_{i}-\frac{3}{2}\right)(i=j)\end{array}\right.$

Where $R_{i j}$ is the distance between the midpoint of elements $i$ and $j$, and $L_{i}$ is the length of element $i$. Therefore, the charge, $q_{i}$, on each element could be calculated by solving the following system of linear algebraic equations.

$G_{i j} q_{i}=v_{j}$

By having the charge and voltage of each element, the total electrical potential energy of the two-dimensional conductors can be calculated using Eq. (5).

$E(x, y)=\frac{1}{2} q^{T} v$

where $q^{T}$ denotes the transpose of $q$. The electrostatic force on each electrode is the gradient of the potential energy multiplied by the length of the moving electrode $(L)$.

$\vec{F}=F_{x}(x, y) \hat{i}+F_{y}(x, y) \hat{j}=\nabla(E(x, y)) \times L$

where $\nabla$ is the gradient operator. For the detailed derivation of the potential energy, one could refer to [38]. We have calculated the potential energy and therefore 


\begin{tabular}{|c|c|c|}
\hline Parameter & Symbol & Value \\
\hline Plate length $(\mu \mathrm{m})$ & - & 1000 \\
Plate width $(\mu \mathrm{m})$ & - & 1000 \\
Moving electrode length $(\mu \mathrm{m})$ & $L$ & 200 \\
Total number of electrode sets & $N$ & 112 \\
Moving electrode width $(\mu \mathrm{m})$ & - & 6 \\
Side electrode width $(\mu \mathrm{m})$ & - & 4 \\
Bottom electrode width $(\mu \mathrm{m})$ & - & 16 \\
Gap between side and bottom & - & 6 \\
electrodes $(\mu \mathrm{m})$ & & \\
Moving electrode thickness $(\mu \mathrm{m})$ & - & 1.5 \\
Bottom and side electrode & & 0.5 \\
thickness $(\mu \mathrm{m})$ & & \\
Initial gap between moving and & $d$ & 2.75 \\
bottom electrodes $(\mu \mathrm{m})$ & & \\
mass of microstructure $(\mathrm{ng})$ & $m$ & 2907 \\
Nominal mechanical stiffness & $k$ & 0.203 \\
$\quad$ N/m) & & \\
Natural frequency $(\mathrm{Hz})$ & $f_{n y}$ & 1330 \\
Time constant $(\mathrm{S})$ & $T_{n}$ & $1 / f_{n y}$ \\
\hline$\quad$ & & \\
\hline$\quad$
\end{tabular}

Table 1: Dimensions for the microstructure in Figure 1.

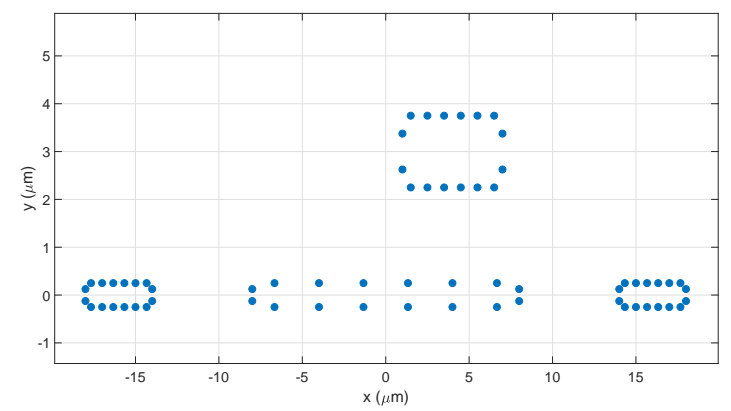

Fig. 5: Discretization of the electrodes surfaces to calculate the potential energy.

the electrostatic force for $-20 \mu m \leq x \leq 20 \mu m$ and $0.1 \mu \mathrm{m} \leq y \leq 25 \mu \mathrm{m}$ with a grid step of $0.1 \mu \mathrm{m}$. Figures 6 and 7 show the electrostatic force surfaces as a function of the position of the moving electrode $(x, y)$ for a part of the considered domain. These figures reveal the nonlinear dependence of the electrostatic force on the position of the moving electrode, making Eq. (15) a set of coupled nonlinear ordinary differential equations. It is worth mentioning that the electrostatic force in the repulsive paradigm can be measured experimentally according to the procedure explained in [39]. In the next section, we investigate the nonlinear dynamics of the system by constructing the Jacobian matrix and performing stability analysis for equilibrium points of the system.

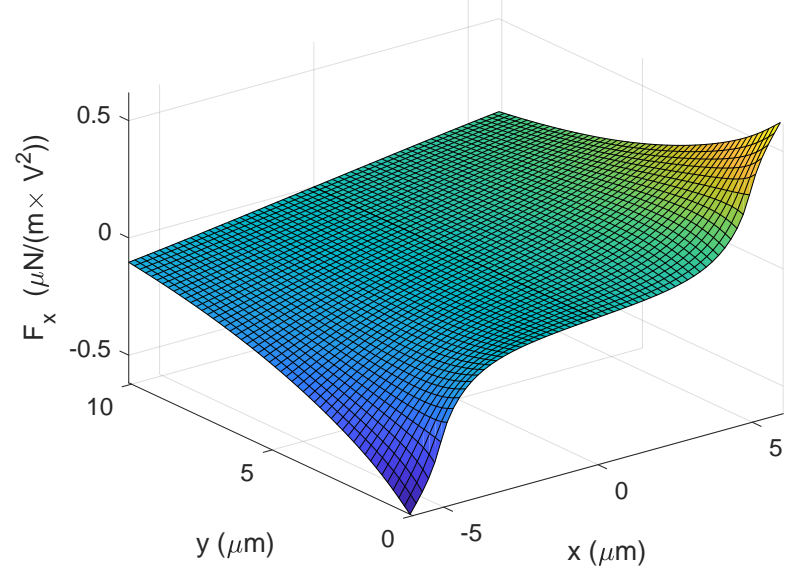

Fig. 6: The lateral component of the electrostatic force $\left(F_{x}(x, y)\right)$. For presentation purposes, the range for $x$ and $y$ are smaller than the range considered to solve Eq. (8).

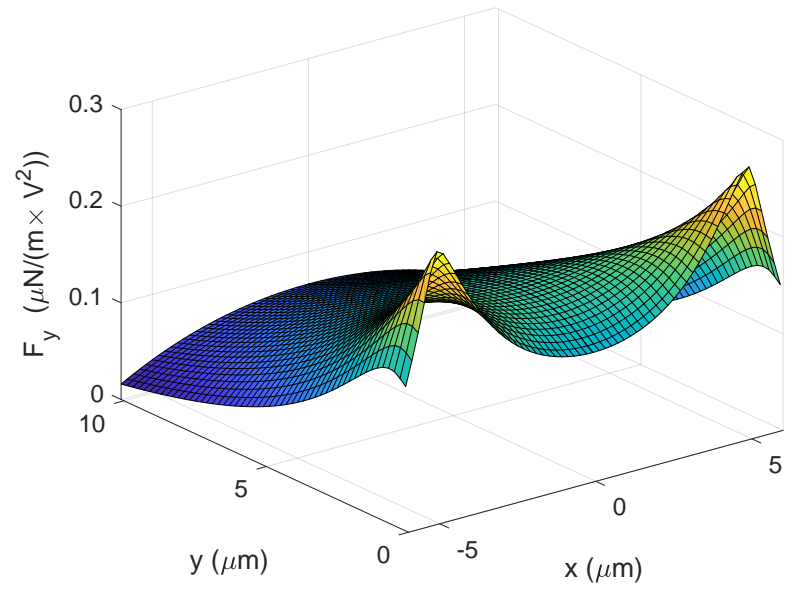

Fig. 7: The transverse component of the electrostatic force $\left(F_{y}(x, y)\right)$. For presentation purposes, the range for $x$ and $y$ are smaller than the range considered to solve Eq. (8).

\section{Stability Analysis and Static Pull-in}

To perform the stability analysis, we first non-dimensionalize Eq. (15) using parameters given in Eq. (7).

$\hat{x}=\frac{x}{d}, \hat{y}=\frac{y}{d}, \hat{t}=\frac{t}{T_{n}}$

Where $d$ and $T_{n}$ are given in Table 1 . Using these parameters to rewrite Eq. (15), and then dropping the hats, we can write: 

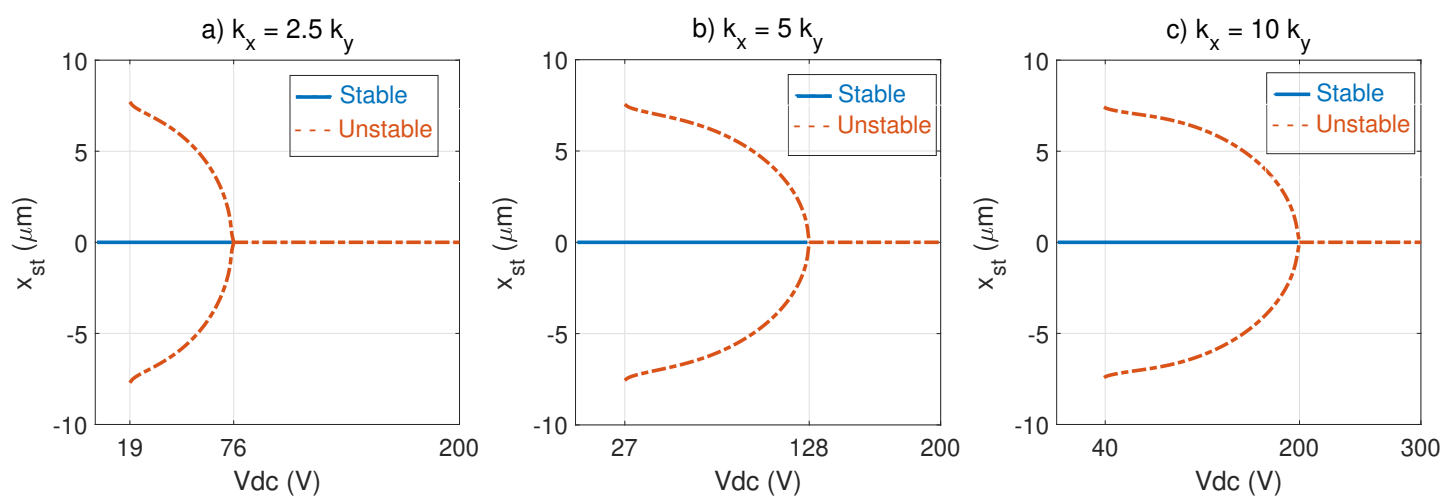

Fig. 8: Bifurcation diagram for the horizontal position of the equilibrium points and their stability for different lateral to transverse stiffness ratios as the voltage on the side electrode changes. The transverse stiffness is kept constant at $k_{y}=0.203(\mathrm{~N} / \mathrm{m})$.
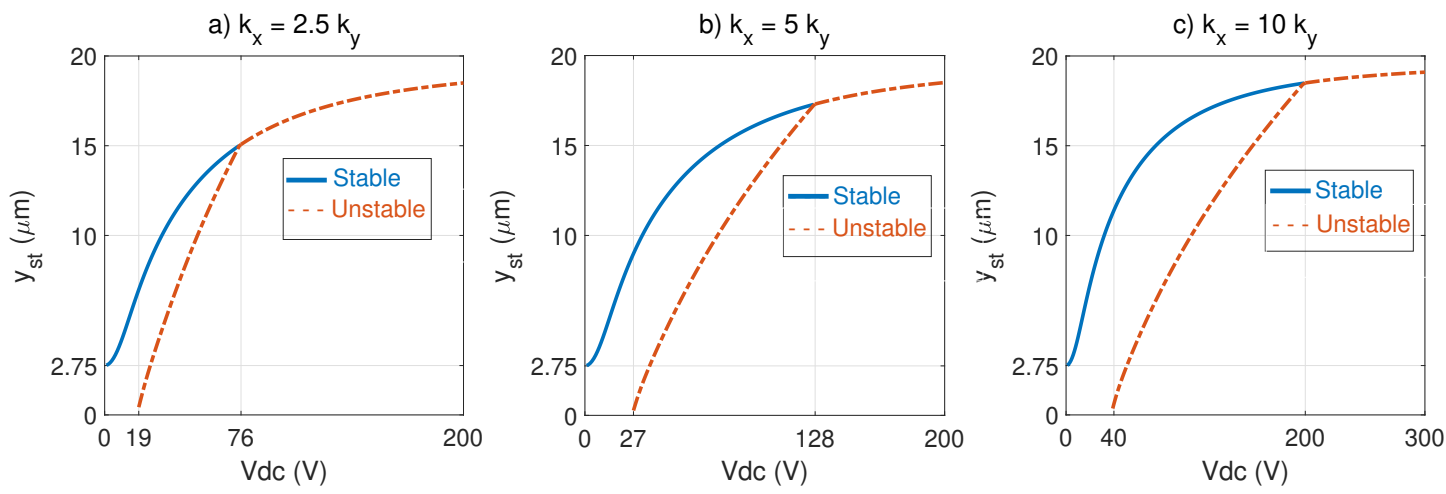

Fig. 9: Bifurcation diagram for the vertical position of the equilibrium points and their stability for different lateral to transverse stiffness ratio as the voltage on side electrode changes. The transverse stiffness is kept constant at $k_{y}=0.203(N / m)$.

$\left\{\begin{array}{l}\ddot{x}+\left(\omega_{x} T_{n}\right)^{2} x=\frac{N T_{n}^{2} V^{2}}{m d} F_{x}(x, y) \\ \ddot{y}+\left(\omega_{y} T_{n}\right)^{2}(y-1)=\frac{N T_{n}^{2} V^{2}}{2 m d} F_{y}(x, y)\end{array}\right.$

To find the equilibrium points of the system, we need to solve the static version of Eq. (8), which is obtained by setting all the time-varying terms equal to zero (Eq. (9)). Equation (9) is a set of two nonlinear algebraic equations that can be solved numerically to yield the equilibrium points of the system, which are denoted by $x_{s t}$ and $y_{s t}$.

$\left\{\begin{array}{l}\left(\omega_{x} T_{n}\right)^{2} x_{s t}=\frac{N T_{n}^{2} V^{2}}{m d} F_{x}\left(x_{s t}, y_{s t}\right) \\ \left(\omega_{y} T_{n}\right)^{2}\left(y_{s t}-1\right)=\frac{N T_{n}^{2} V^{2}}{2 m d} F_{y}\left(x_{s t}, y_{s t}\right)\end{array}\right.$
Equation $((8))$ can be rewritten in the state space using the following variables.

$$
\begin{aligned}
& \left\{\begin{array}{l}
x=x_{1} \\
\dot{x}=x_{2} \\
y=x_{3} \\
\dot{y}=x_{4}
\end{array}\right. \\
& \left\{\begin{array}{l}
\dot{x}_{1}=x_{2}=f_{1}\left(x_{1}, x_{2}, x_{3}, x_{4}\right) \\
\dot{x}_{2}=-\left(\omega_{x} T_{n}\right)^{2} x_{1}+\frac{N T_{n}^{2} V^{2}}{m d} F_{x}\left(x_{1}, x_{3}\right)=f_{2}\left(x_{1}, x_{2}, x_{3}, x_{4}\right) \\
\dot{x}_{3}=x_{4}=f_{3}\left(x_{1}, x_{2}, x_{3}, x_{4}\right) \\
\dot{x}_{4}=-\left(\omega_{y} T_{n}\right)^{2} x_{3}+\frac{N T_{n}^{2} V^{2}}{2 m d} f_{y}\left(x_{1}, x_{3}\right)=f_{4}\left(x_{1}, x_{2}, x_{3}, x_{4}\right)
\end{array}\right.
\end{aligned}
$$



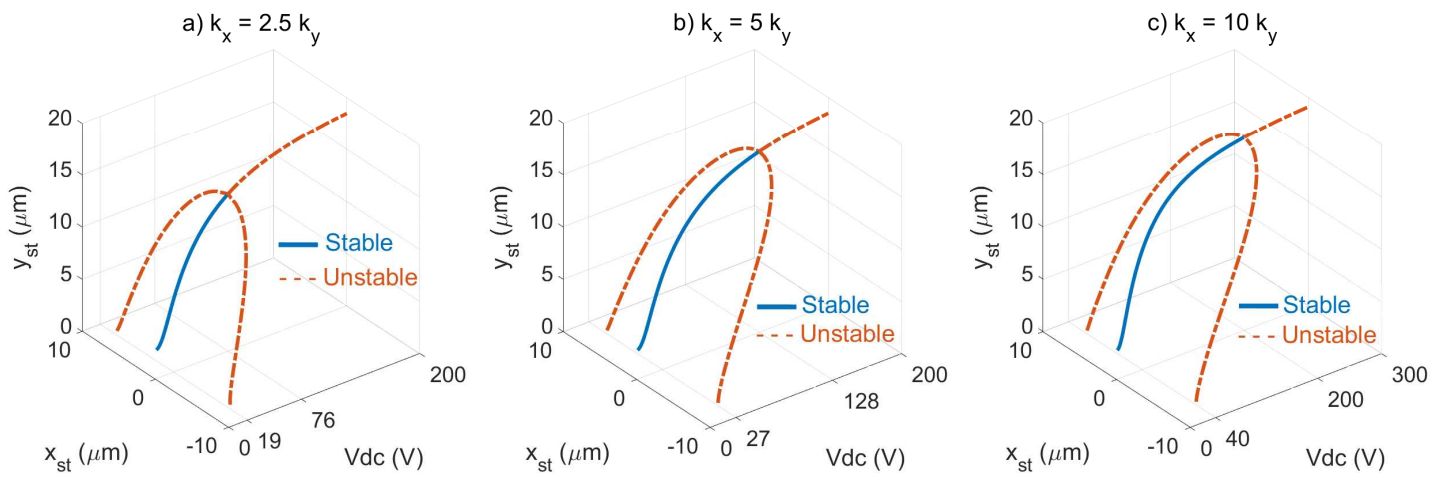

Fig. 10: The 3D bifurcation diagram for different lateral to transverse stiffness ratios. The transverse stiffness is kept constant at $k_{y}=0.203(\mathrm{~N} / \mathrm{m})$.

Assuming the static solution of the system at voltage $V_{d c}$ to be $\left(x_{1}^{*}, x_{3}^{*}\right)$, then we can construct the Jacobian matrix as

$J=\left[\begin{array}{llll}\frac{\partial f_{1}}{x_{1}} & \frac{\partial f_{1}}{x_{2}} & \frac{\partial f_{1}}{x_{3}} & \frac{\partial f_{1}}{x_{4}} \\ \frac{\partial f_{2}}{x_{1}} & \frac{\partial f_{2}}{x_{2}} & \frac{\partial f_{2}}{x_{3}} & \frac{\partial f_{2}}{x_{4}} \\ \frac{\partial f_{3}}{x_{1}} & \frac{\partial f_{3}}{x_{2}} & \frac{\partial f_{3}}{x_{3}} & \frac{\partial f_{3}}{x_{4}} \\ \frac{\partial f_{4}}{x_{1}} & \frac{\partial f_{4}}{x_{2}} & \frac{\partial f_{4}}{x_{3}} & \frac{\partial f_{4}}{x_{4}}\end{array}\right]$

By solving the eigenvalue problem for this Jacobian matrix, we can perform the stability analysis,

$|J-\lambda I|=0$

Where I is the unity matrix.

$\lambda^{4}+\left(\left(\omega_{x} T_{n}\right)^{2}+\left(\omega_{y} T_{n}\right)^{2}-\frac{N T_{n}^{2} V^{2}}{2 m d}\left(\frac{\partial F_{x}}{\partial x}+2 \frac{\partial F_{y}}{\partial y}\right)\right) \lambda^{2}$

$-\frac{N T_{n}^{2} V^{2}}{2 m d}\left(2\left(\omega_{x} T_{n}\right)^{2} \frac{\partial F_{y}}{\partial y}+\left(\omega_{y} T_{n}\right)^{2} \frac{\partial F_{x}}{\partial x}\right)$

$+\left(\frac{N T_{n}^{2} V^{2}}{2 m d}\right)^{2}\left(2 \frac{\partial F_{x}}{\partial x} \frac{\partial F_{y}}{\partial y}-2 \frac{\partial F_{x}}{\partial y} \frac{\partial F_{y}}{\partial x}\right)+\left(\omega_{x} \omega_{y}\right)^{2} T_{n}^{4}=0$

Equation (14) is a quadratic equation in $\lambda^{2}$, that can be solved using the quadratic formula for the roots of a second-degree algebraic equation. For each case of $k_{x}$ and $k_{y}$, we calculate the eigenvalues at each equilibrium point for a range of voltages. If the real parts of all the four eigenvalues are smaller than or equal to zero, the equilibrium point is stable (or marginally stable). Otherwise, the equilibrium point is considered to be unstable.

To illustrate the stability of the equilibrium points the bifurcation diagrams with the control parameter of DC voltage are depicted in Figs. 8, 9, and 10. In Fig. 8 (a), the lateral stiffness is chosen to be 2.5 times larger than the transverse stiffness. This figure shows that for the small voltages up to $19(V)$ there is only one equilibrium position for the system, which is stable. This stable point is at the center position where the electrostatic force from the right-side electrode is equal to the force from the left-side electrode. As the voltage is increased, two unstable equilibrium points appear on both sides of the center position. By increasing the voltage, these two unstable equilibrium positions get closer to the center position. The existence of these two unstable side solutions means that if the microstructure is subjected to a lateral displacement from an unwanted load such as the mechanical shock load, the microstructure might collapse because of pull-in. This establishes a lateral shock load threshold for microstructures built on the repulsive electrode sets that should be considered by the MEMS designer. By continuing to increase the voltage, these two side branches eventually merge with the stable equilibrium point at the center, making it unstable through a subcritical pitchfork bifurcation. Beyond this point $(76(V))$, there is no stable equilibrium position for the system, which means that the microstructure is going to be pulled-in to the side electrodes. This voltage limit establishes another design criterion for microstructures based on the repulsive electrode sets, limiting the maximum allowed voltage for them. It is worth mentioning that the dynamic pull-in might still happen at lower voltages, but this study focuses only on the static pull-in. 

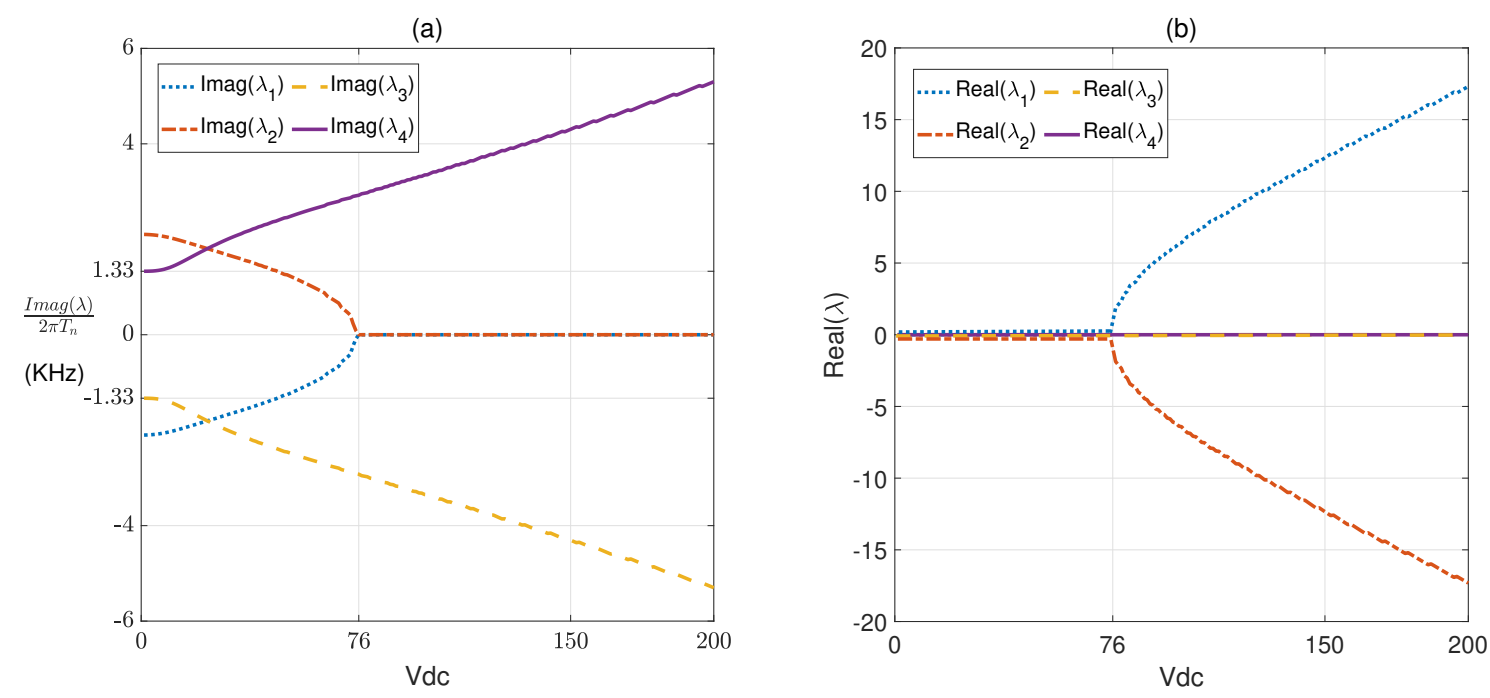

Fig. 11: Imaginary (a) and real (b) parts of the eigenvalues for the equilibrium points at the center, where $x_{s t}$ is zero $\left(k_{y}=0.203(N / m)\right.$ and $\left.k_{x}=2.5 k_{y}\right)$. The real parts of all the eigenvalues are zero up to the bifurcation point $\left(V_{d c}=76(V)\right)$. The imaginary parts of two of the eigenvalues, $\left(\lambda_{1}\right.$ and $\left.\lambda_{2}\right)$ go to zero at the bifurcation point. After bifurcation happens, the real part of $\lambda_{1}$ becomes a nonzero positive number, indicating that the equilibrium point at the center turns to a saddle point and is unstable.

(a)

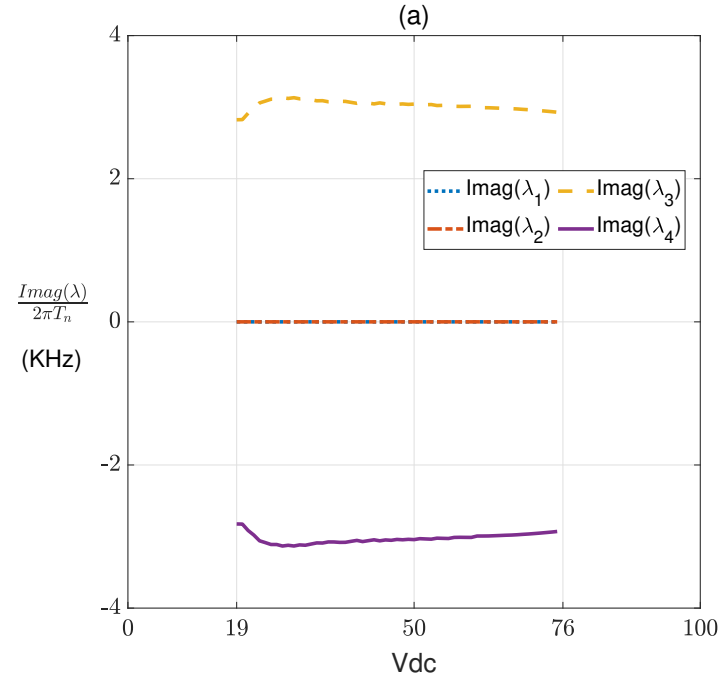

(b)

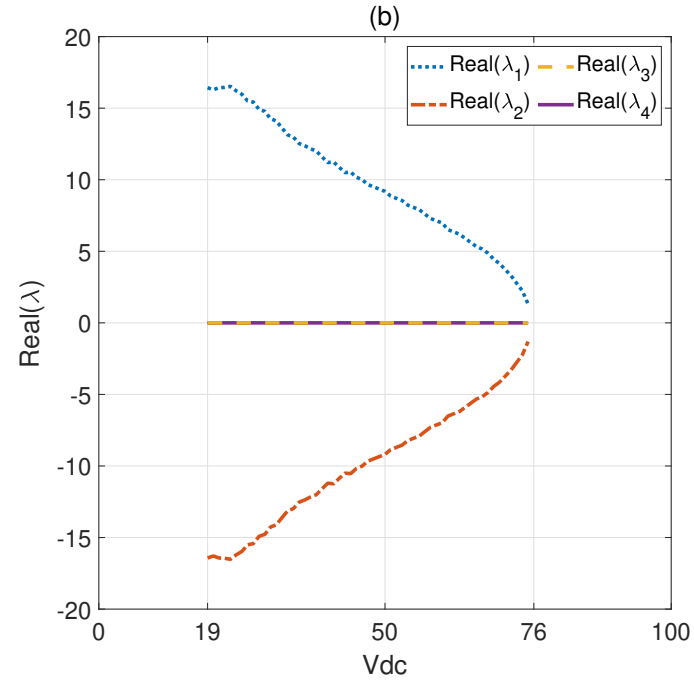

Fig. 12: Imaginary (a) and real (b) parts of the eigenvalues for the equilibrium points on the side of the center position, where $x_{s t}$ is not zero $\left(k_{y}=0.203(\mathrm{~N} / \mathrm{m})\right.$ and $\left.k_{x}=2.5 k_{y}\right)$. The real part of $\lambda_{1}$ has a positive value, which means the side equilibrium points are unstable. At bifurcation point $(76 \mathrm{~V})$, two of the eigenvalues go to zero as the side solutions merge with the stable solution at the center, destroying its stability.

Figure 11 shows the imaginary and real parts of the eigenvalues for the equilibrium points at the center $\left(x_{s t}=0\right)$. At the bifurcation point, the imaginary parts of the eigenvalues, which represent linearized natural 
frequencies, go to zero. The real parts of all the eigenvalues are zero up to the bifurcation point, where the voltage on the side electrodes is $76(\mathrm{~V})$. Therefore, the Jacobian matrix has a zero eigenvalue at $76(\mathrm{~V})$ which is a sign for bifurcation [25]. The real part of $\lambda_{1}$ becomes a nonzero positive number after bifurcation happens, which means the equilibrium point at the center turns to a saddle point and is unstable. This qualitative change in the behavior of the system is another sign for bifurcation [25]. Figure 12 shows the real and imaginary parts of the eigenvalues for the equilibrium points on the side of the center position, where $x_{s t}$ is not zero. The real part of $\lambda_{1}$ has a positive value, which means the side equilibrium points are unstable. At bifurcation point $(76 \mathrm{~V})$, two of the eigenvalues go to zero as the side solutions merge with the stable solution at the center, destroying its stability.

Figure 8 (b) and (c) display the horizontal position of the stable and unstable equilibrium points when the lateral stiffness is 5 and 10 times larger than the transverse stiffness respectively. These figures reveal that by increasing the lateral stiffness, the bifurcation can be postponed to higher voltages. Especially, they suggest that if the microstructure is designed to sense or actuate only in the transverse direction, it is better to have the lateral stiffness to be larger so that the device can operate even at higher voltages without pull-in failure. However, increasing the lateral stiffness might not always be a safe design option in terms of providing flexible supports that allow for the release of residual fabrication stress in the plate without causing the plate to buckle. So, for each microstructure based on the application, the fabrication process, and the expected per-

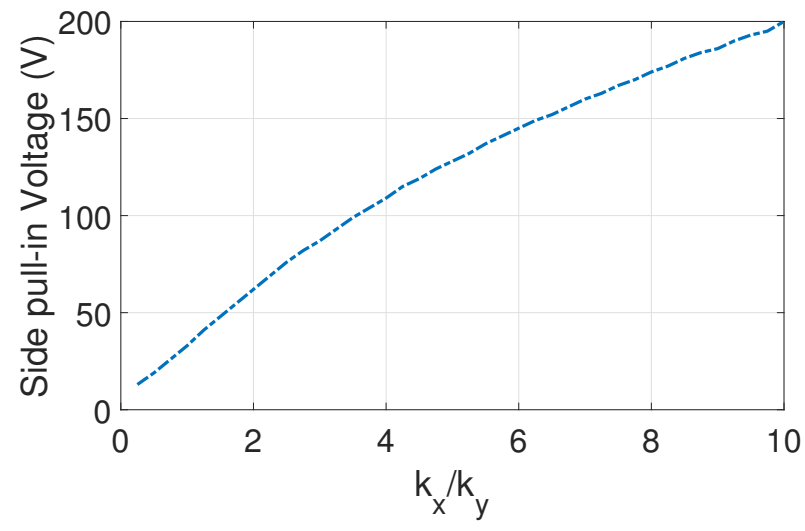

Fig. 13: The pull-in voltage for different ratios of lateral to transverse stiffness. At the given transverse stiffness, beyond the bifurcation point there is no stable equilibrium point for the microstructure. The transverse stiffness is kept constant at $k_{y}=0.203(\mathrm{~N} / \mathrm{m})$. formance from the device, this bifurcation point should be considered along with other design criteria to build a robust and reliable MEMS device. Figure 9 shows the bifurcation diagram for the vertical position of the stable and unstable equilibrium points as the voltage on the side electrode changes for different stiffness scenarios. Because the mathematical model of the system has two DOFs, the real bifurcation diagram for the system is a three-dimensional (3D) graph that shows how the horizontal and vertical positions of the equilibrium points change with the control parameter, voltage $(V)$. Figure 10 shows the 3D bifurcation diagrams for each stiffness scenario.

Because the bifurcation point determines the maximum allowed voltage for the microstructure, it is important to know its variation with the ratio of the lateral and transverse stiffness, see Fig. 13.

The bifurcation threshold's dependence on the value of transverse stiffness is not intuitive. For instance, for a constant lateral stiffness $\left(k_{x}\right)$, the bifurcation threshold would actually decrease with an increase in the transverse stiffness $\left(k_{y}\right)$. By increasing the transverse stiffness, the vertical components of the equilibrium points $\left(y_{s t}\right)$ will decrease. This is because the restoring force of the microstructure is able to hold the microstructure at lower gaps where the net vertical component of the electrostatic force $\left(F_{y}\right)$ is larger. On the other hand, because the horizontal component of the electrostatic force $\left(F_{x}\right)$ is larger at lower vertical gaps (see Fig. 6), the horizontal stiffness of the microstructure would not be able to resist the electrostatic force in the lateral direction.

To further illustrate this, the static equation is solved for three different transverse stiffnesses. The results for the stability analysis of the equilibrium points are shown in figures 14 and 15. Figure 14 shows that for the extreme case where the transverse stiffness is zero the bifurcation point, beyond which there is no stable solution for the microstructure, happens at $110(V)$. However, by increasing the transverse stiffness to 10 times larger than the lateral stiffness, this bifurcation point happens at the lower voltage of $40(\mathrm{~V})$. In another extreme case where the lateral stiffness is significantly larger than the horizontal stiffness (1000 times larger) (Fig. 14) (c), the system exhibits another bifurcation, a saddle-node bifurcation. IN this case, there are 5 equilibrium points for the system at certain levels of voltage. Two of these Equilibrium points are unstable while 3 of them are stable. It is interesting to notice that the center horizontal position becomes unstable at $41(V)$ even though 

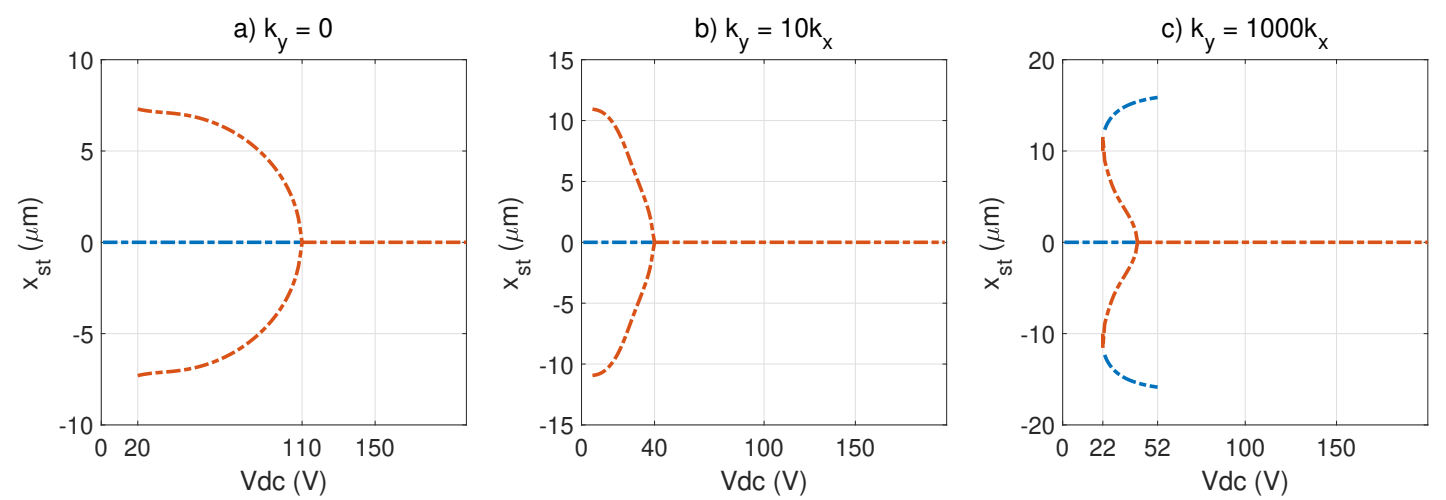

Fig. 14: Bifurcation diagram for the horizontal position of the equilibrium points for different transverse to lateral stiffness ratios as the voltage on side electrode changes. The lateral stiffness is kept constant at $2.5 \mathrm{k}$.
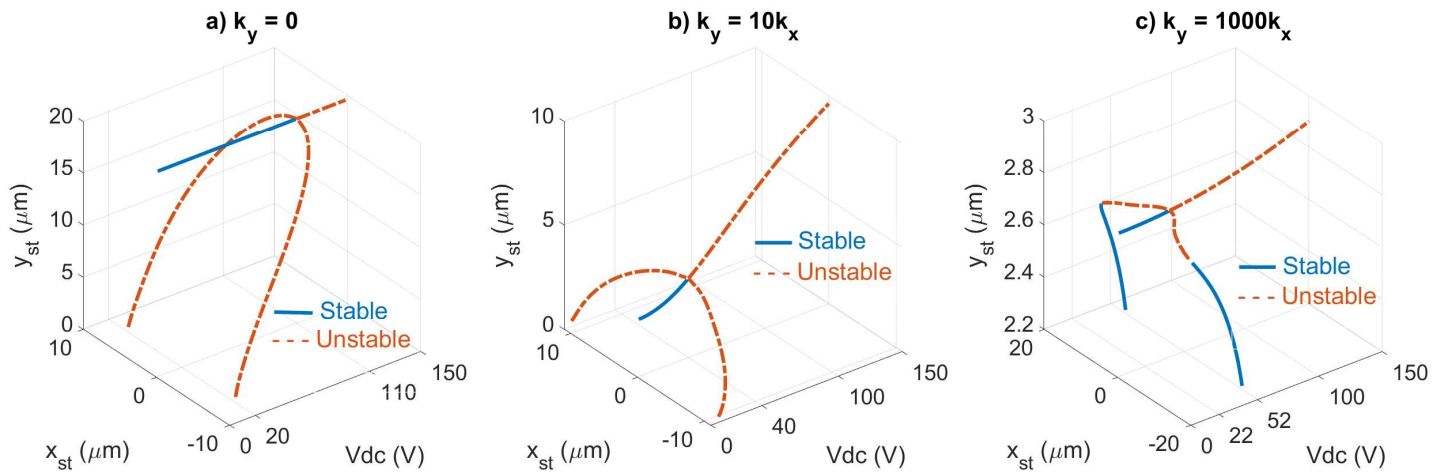

Fig. 15: The 3D bifurcation diagram for different lateral to transverse stiffness ratios. The lateral stiffness is kept constant at $2.5 \mathrm{k}$.

the transverse stiffness is 100 times larger compared to the transverse stiffness in Fig. 14 (b). However, for the higher stiffness case there are two stable side solutions up to $52(V)$, beyond which there is no stable equilibrium point.

Figure 15 shows the 3D stability diagrams for the three cases shown in Fig. 14, illustrating subcritical pitchfork and saddle-node bifurcations. This figure shows when the transverse stiffness is zero, the microstructure tends to go to the vertical position at the center where the vertical component of the electrostatic force is zero. When the transverse stiffness is significantly large, the microstructure stays close to its initial fabrication gap.

\section{The effect of Fabrication Imperfection}

In this section, we have investigated the effect of an initial lateral misplacement for the moving electrode on the pull-in voltage. Such a misplacement might happen because of fabrication imperfections. Figure 16 shows the initial position of the moving electrode off the center position by $\delta$.

In this case, the governing equation of motion can be written as follows:

$\left\{\begin{array}{l}m \ddot{x}+k_{x}(x-\delta)=\left(\frac{N}{2}\right) V^{2} F_{x}(x, y) \\ m \ddot{y}+k_{y}(y-d)=(N) V^{2} F_{y}(x, y)\end{array}\right.$

Performing the stability analysis similar to that of section 5, the equilibrium points can be extracted for the voltages on the side electrodes. Figure 17 shows the 


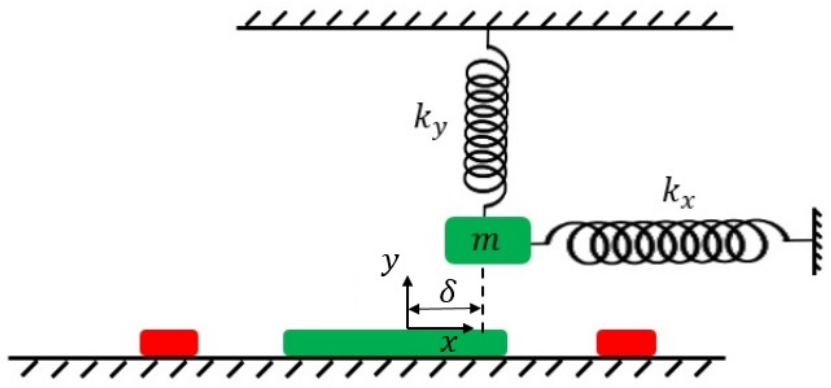

Fig. 16: The two DOF mathematical model of the microstructure when the moving electrode is not at the center position.

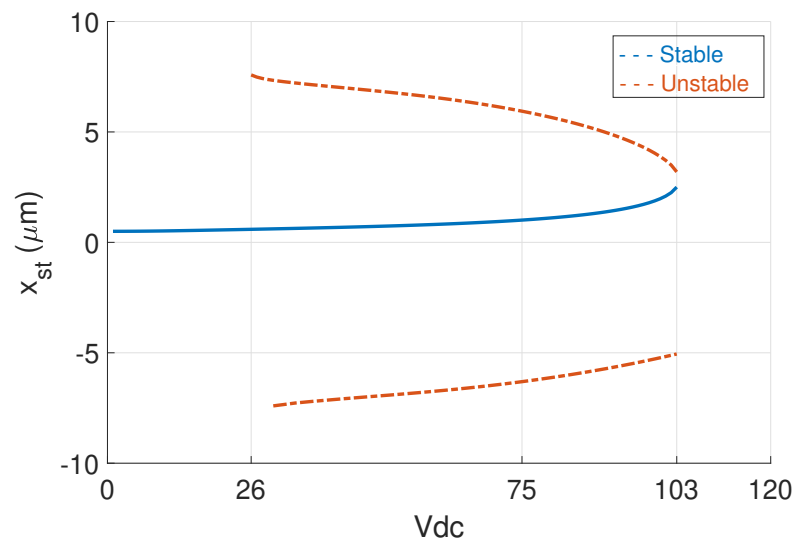

Fig. 17: Bifurcation diagram for the horizontal position of the equilibrium points when $\delta=0.5 \mu \mathrm{m}$. The transverse and lateral stuffiness are $k_{y}=0.203(\mathrm{~N} / \mathrm{m})$ and $k_{x}=5 k_{y}$ respectively.

$\mathrm{x}$ components of the equilibrium points when the moving electrode is initially misplaced by $0.5 \mu \mathrm{m}$ in the lateral direction. Comparing this figure with the results for when the moving electrode is at center (Fig. 8) shows that the subcritical pitchfork bifurcation is replaced by a saddle-node bifurcation. The stable and unstable solutions destroy each other at $103(V)$. Beyond this voltage, there is no equilibrium point for the microstrucutre. This bifurcation point establishes the pull-in voltage for the microstructure. Figure 18 shows how the saddlenode bifurcation point depends on the stiffness ratio for different initial misplacements. As illustrated in this figure, the pull-in threshold will decrease noticeably by increasing the initial misplacement, $\delta$.

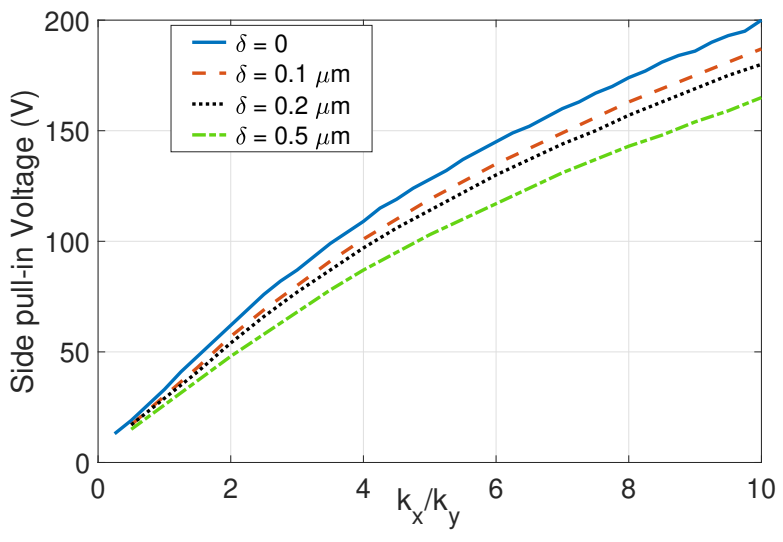

Fig. 18: The effect of lateral misplacement on the pullin voltage for different ratios of lateral to transverse stiffness. The transverse stiffness is kept constant at $k_{y}=0.203(N / m)$.

\section{Conclusion}

The lateral instability of electrostatic MEMS devices based on the repulsive force is reported. The instability mode is explained qualitatively in terms of static pull-in of the microstructure. A two degree-of-freedom mathematical model is developed to investigate the pull-in of the microstructure. The results from the stability analysis of the model show the existence of pull-in instability for electrostatic transducers that utilize the repulsive force. Different stiffness scenarios are discussed and the bifurcation diagrams are extracted from analyzing the eigenvalues of the Jacobian matrix. It is shown that the ratio between the lateral to transverse stiffness is a very important design parameter for building reliable and robust transducers based on the the repulsive scheme. This study shows that the voltage on the side electrodes cannot be increased without limitation. Depending on the ratio of the stiffness, there is a voltage limit beyond which there is no stable equilibrium point for the microstructure. This will impose a limit for maximum voltage that is allowed for the microstructure without jeopardizing the its structural integrity. The effect of fabrication imperfection is analyzed by initially displacing the moving electrode from the center position. The results show a noticeable decrease in the pull-in threshold as a result of such imperfection.

\section{Acknowledgment}

The authors would like to acknowledge the financial support of this study by the National Science Foundation (NSF) through grant ECCS 1608692. 


\section{Conflict of Interest}

The authors declare that they have no conflict of interest.

\section{References}

1. Guney, M. G., Li, X. , Chung, V. P. J., paramesh, J., Mukherjee, T., Fedder, G. K., High dynamic range CMOSMEMS capacitive accelerometer array, 2018 IEEE Micro Electro Mechanical Systems (MEMS), pp. 992-995. (2018).

2. R. Mukhiya, P. Agarwal, S. Badjatya, M. Garg, P. Gaikwad, S. Sinha, A. K. Singh, and R. Gopal, 2019." Design, modelling and system level simulations of DRIE-based MEMS differential capacitive accelerometer".Microsystem Technologies, 25 (9), pp. 3521-3532, (2019).

3. Yang, C., Tang, S., and Tavassolian, Utilizing Gyroscopes Towards the Automatic Annotation of Seismocardiograms, IEEE Sensors Journal, Vol. 17(7), pp. 2129-2136, (2017).

4. Sheikhaleh, A., Jafari, K., and Abedi K., Design and Analysis of a Novel MOEMS Gyroscope Using an Electrostatic Comb-Drive Actuator and an Optical Sensing System, IEEE Sensors Journal, 19(1), pp. 144-150, (2019).

5. Pallay, M. and Towfighian, S., A Combined MEMS Threshold Pressure Sensor and Switch, IEEE Sensors Conference, (2019).

6. Wang, Z., Zhang, Q., Wang, W., and Han, J., Dynamic Analysis of a Micro Beam-Based Tactile Sensor Actuated by Fringing Electrostatic Fields, Micromachines, Vol. 10(5), (2019).

7. Miles, R. N., Cui, W., Su, Q. T., and Homentcovschi, D., A MEMS Low-Noise Sound Pressure Gradient Microphone With Capacitive Sensing, Journal of Microelectromechanical Systems, 24(1), pp. 241-248, (2015).

8. Ozdogan, M., Towfighian, S., and Miles, R. N., Fabrication and Experimental Characterization of a MEMS Microphone Using Electrostatic Levitation, 2019 IEEE Sensors Conference, (2019).

9. Pallay, M. and Towfighian, S., A reliable MEMS switch using electrostatic levitation, Applied Physics Letters, Vol. 113, (2018).

10. Jacopo Iannacci, Reliability of MEMS: A perspective on failure mechanisms, improvement solutions and best practices at development level, Displays, vol 37, pp. 62-71 (2014).

11. Ramini, A., Bellaredj, M. L. F., Hafiz, M. A. A., and Younis, M. I., Experimental investigation of snap-through motion of in-plane MEMS shallow arches under electrostatic excitation, Journal of Micromechanics and Microengineering, Vol. 26(1), (2015).

12. Derakhshani, M and Berfield, T. A., Snap-Through and Mechanical Strain Analysis of a MEMS Bistable Vibration Energy Harvester, Shock and Vibration (2019).

13. Maani Miandoab, E., Nejat Pishkenari, H., Meghdari, A., and Fathi, M., A general closed-form solution for the static pull-in voltages of electrostatically actuated MEMS/NEMS, Physica E: Low-dimensional Systems and Nanostructures, Vol. 90, pp. 7-12, (2017).

14. SoltanRezaee, M. and Afrashi, M., Modeling the nonlinear pull-in behavior of tunable nano-switches, International Journal of Engineering Science, Vol. 109, pp. 73-87, (2016).

15. Ozdogan, M., Towfighian, S., and Miles, R. N., Modeling and Characterization of A Pull-in Free MEMS Microphone, IEEE Sensors Journal, (2020).
16. Godara, R. K., and Joglekar M. M., Alleviation of residual oscillations in electrostatically actuated variable-width microbeams using a feedforward control strategy, Microsystem Technologies, Vol 23, (2016).

17. Godara, R. K., and Joglekar M. M., Suppression of contact bounce in beam-type microelectromechanical switches using a feedforward control scheme, Journal of Vibration and Control, (2018).

18. Lee, K. B., and Cho, Y., Laterally driven electrostatic repulsive-force microactuators using asymmetric field distribution, Journal of Microelectromechanical Systems, Vol. 10(1), pp. 128-136, (2001).

19. Towfighian, S., Seleim, A., Abdel-Rahman, E. M., and Heppler, G. R., A Large-Stroke Electrostatic MicroActuator, Journal of Micromechanics and Microengineering, Vol. 21(7), (2011).

20. Park, S., Khater, M., Effa, D., Abdel-Rahman, E., and Yavuz, M., Detection of cyclic-fold bifurcation in electrostatic MEMS transducers by motion-induced current, Journal of Micromechanics and Microengineering, Vol. 27(8), (2017).

21. Ak, C., and Yildiz, A., A Novel Closed-Form Expression Obtained by Using Differential Evolution Algorithm to Calculate Pull-In Voltage of MEMS Cantilever, Journal of Microelectromechanical Systems, Vol. 27(3), pp. 392-397, (2018).

22. Zehnder, A. T., Rand, R. H., and Krylov, S., Locking of electrostatically coupled thermo-optically driven MEMS limit cycle oscillators, International Journal of Non-Linear Mechanics, vol. 102, pp. 92-100 (2018).

23. Caruntu, D. I., Botello, M. A., Reyes, C. A., Beatriz, J. S., Voltage-Amplitude Response of Superharmonic Resonance of Second Order of Electrostatically Actuated MEMS Cantilever Resonators, Journal of Computational and Nonlinear Dynamics, Vol. 14, (2019).

24. Guha, K. and Laskar, N. M. Gogoi, H. J. Chanda, S. Baishnab, K. L., Rao, K. S., and Maity, N. P., An improved analytical model for static pull-in voltage of a flexured MEMS switch, Microsystem Technologies, (2018).

25. Younis, Mohammad I., MEMS Linear and Nonlinear Statics and Dynamics, Springer, New York (2011).

26. Zhang, W., Yan, H., and Peng, Z., and Meng, G., Electrostatic pull-in instability in MEMS/NEMS: A review, Sensors and Actuators A: Physical, vol. 214, pp. 187-218 (2014).

27. Bian, W., Zhao, J., and You, Z., Low voltage, high speed and small area in-plane MEMS switch, Journal of Micromechanics and Microengineering, Vol. 29(6), (2019).

28. Firouzi, B., and Zamanian, M., The effect of capillary and intermolecular forces on instability of the electrostatically actuated microbeam with T-shaped paddle in the presence of fringing field, Applied Mathematical Modeling, Vol. 71, pp. 243-268 (2019).

29. Sharma, A. K., Godara, R. K., and Joglekar, M. M., Static and DC dynamic pull-in analysis of curled microcantilevers with a compliant support, Microsystem Technologies, Vol. 25(3), pp. 965-975 (2019) .

30. Nayfeh, Ali H., Younis, M. I., and Eihab, A. M., Dynamic pull-in phenomenon in MEMS resonators, Nonlinear Dynamics, Vol. 48, No. 1 (2007).

31. Rocha, L. A., Cretu, E., and Wolffenbuttel, R. F., Behavioural analysis of the pull-in dynamic transition, Journal of Micromechanics and Microengineering, Vol. 14, pp.37-42, (2004).

32. Sharma, M., Sarraf, E. H., and Cretu, E., A novel dynamic pull-in MEMS gyroscope, Procedia Engineering, Vol. 25, pp. 55-58, (2011). 
33. pallay, M., Daeichin, M., and Towfighian, S., Dynamic behavior of an electrostatic MEMS resonator with repulsive actuation. Nonlinear Dynamics, 89(2), (2017).

34. Pallay, M. and Towfighian, S., Feasibility Study of a Capacitive MEMS Filter Using Electrostatic Levitation, International Design Engineering Technical Conferences, (2019).

35. Daeichin, M., Ozdogan, M., Towfighian, S., and Miles, R. N., Dynamic Response of a Tunable MEMS Accelerometer Based on Repulsive Force, Sensors and Actuators: A. Physical (2019).

36. Parametric resonance of a repulsive force MEMS electrostatic mirror, Ozdogan, M., Daeichin, M., Ramini, A., and Towfighian, S., Sensors and Actuators A: Physical, vol. 265, (2017).

37. Miles, R. N., A Compliant Capacitive Sensor for Acoustics: Avoiding Electrostatic Forces at High Bias Voltages, IEEE Sensors Journal, 18(14), pp. 5691-5698, (2018).

38. Miles, R. N., Physical Approach to Engineering Acoustics, Springer, New York (2019).

39. Daeichin, M., Miles, R. N., Towfighian, S., Experimental characterization of the electrostatic levitation force in MEMS transducers, Journal of Vibration and Acoustics, (2020). 